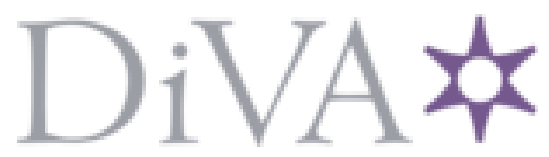

http://www.diva-portal.org

This is the published version of a paper published in Behavioural and Cognitive Psychotherapy.

Citation for the original published paper (version of record):

Norell Clarke, A., Nyander, E., Jansson-Fröjmark, M. (2011)

Sleepless in Sweden: a single subject study of effects of cognitive therapy for insomnia on three adolescents.

Behavioural and Cognitive Psychotherapy, 39: 367-374

http://dx.doi.org/10.1017/S1352465810000664

Access to the published version may require subscription.

N.B. When citing this work, cite the original published paper.

Permanent link to this version:

http://urn.kb.se/resolve?urn=urn:nbn:se:oru:diva-32221 


\title{
Sleepless in Sweden: A Single Subject Study of Effects of Cognitive Therapy for Insomnia on Three Adolescents
}

\author{
Annika Norell-Clarke, EvaLotta Nyander and Markus Jansson-Fröjmark
}

Örebro University, Sweden

\begin{abstract}
Background: Sleeping difficulties are an increasing problem for youths, but there is a lack of treatment research for this age group. Aim: The aim of this study was to investigate the effects of Cognitive Therapy for Insomnia (CT-I) on youths with primary insomnia; this treatment has never been tested on youths before. Method: The study was conducted according to an $\mathrm{AB}$ single-case experimental phase design, with pre-tests and post-tests. After 7-10 days of baseline assessment, three youths aged 16-18 (2 male) with primary insomnia participated in a 7-week long treatment. A sleep diary was used throughout the treatment. A followup assessment including one week with a sleep diary was conducted 3 months later. Visual inspection was used to analyze outcome. Results: Insomnia severity was greatly reduced for all participants after treatment. Daily measures showed that sleep onset latency was reduced for two participants but no change in total sleep time was confirmed. Daytime symptoms fluctuated for the participants. The insomnia-specific psychological processes were reduced to varying extents. These results were maintained at the follow-up measure. Conclusions: CT-I may be a promising treatment for youths with insomnia and the treatment should be tested further in randomized controlled studies.
\end{abstract}

Keywords: Insomnia, cognitive therapy, single-case, adolescence.

\section{Introduction}

Many adolescents of today sleep poorly. The most common sleep disorder, insomnia, means difficulties in falling asleep, staying asleep, or not feeling rested in the morning for at least one month combined with daytime symptoms. Between 5 and $16 \%$ of adolescents fulfil criteria of insomnia, with up to $25 \%$ reporting at least one symptom of insomnia (e.g. Roberts, Roberts and Duong, 2008). Adolescents who do not get enough sleep are more likely to develop problems with their somatic or psychological health (Roberts et al., 2008) and they run a higher risk of developing depression (Breslau, Roth, Rosenthal and Andreski, 1996). Yet there have been few attempts to treat adolescents with poor sleep by using psychological methods and there is a need for more research on adolescents.

Cognitive behavioural therapy is an evidence-based treatment for adult insomnia (Morin et al., 2006), but adolescent sleep is affected by specific developmental and lifestyle factors so

Reprint requests to Annika Norell-Clarke, Örebro University, School of Law, Psychology and Social Work, Fakultetsgatan 1, Örebro 70182, Sweden. E-mail: annika.norell@oru.se An extended version is also available online in the table of contents for this issue: http://journals.cambridge.org/jid_BCP 
it cannot be concluded that treatments tested on adults would also be effective for adolescents. When psychological methods have been tested on youth with sleep problems, the study has either been school interventions aimed at participants without a sleep disorder diagnosis (Moseley and Gradisar, 2009; Sousa, Araújo and Azevedo, 2007), or treatment for insomnia comorbid with drug addiction (Bootzin and Stevens, 2005). Treatments for adolescents with primary insomnia have yet to be studied.

Recent research has emphasized the role of cognitive processes in insomnia. Five cognitive processes are theorized to maintain chronic insomnia by being present during both day and night and by reinforcing each other: worry; selective attention and monitoring; misperception; unhelpful beliefs about sleep and consequences of bad sleep; and safety behaviours. For a more detailed description see Harvey, 2005. A newly developed treatment, Cognitive Therapy for Insomnia, (CT-I) targets these maintaining processes and was efficient in an open trial with adult participants (Harvey, Sharpley, Ree, Stinson and Clark, 2007).

The purpose of this study was to test a cognitive insomnia treatment on adolescents aged (16-18) by replicating a previous study with cognitive therapy for insomnia (Harvey et al., 2007). Single case experimental designs, as used in this study, have the advantage of offering a carefully controlled investigation on a small scale, which is suitable when evaluating new treatments. Daily measures of target variables during a baseline phase and a treatment phase means that threats to internal validity can be ruled out, as every individual serves as their own control. If improvements of target variables occur only after the treatment phase has started, these changes are assumed to be caused by the treatment.

\section{Method}

\section{Design}

Following a baseline measurement of 7-10 days (phase A) the participants received a 7-week manual based CT-I (phase B). A sleep diary with sleep and daytime items was used throughout treatment. Assessments with validated self-report questionnaires on insomnia severity and cognitive processes before baseline and after treatment were added to the single subject design. A follow-up measurement was conducted 3 months after the treatment was finished and consisted of a sleep diary and the same battery of questionnaires.

\section{Participants}

Participants were recruited from a local upper secondary school through e-mails that were sent to all 475 pupils from the school health care unit. The inclusion criteria were: (a) pupil in upper secondary level (age between 16 and 18 years); (b) meeting DSM-IV-TR criteria for primary insomnia; and (c) sufficient proficiency in Swedish. The exclusion criteria were: (a) evidence that the insomnia could be accounted for by another sleep disorder or psychiatric disorder; (b) evidence of a medical/physical condition that could account for the poor sleep; and (c) receiving other treatment for insomnia. Seven pupils responded to the recruitment efforts and six of them participated in a screening interview. Five participants fulfilled the inclusion criteria, (two boys and three girls from 16 to 18 years old) and started the treatment. Two dropped out during the second week due to competing commitments. 
Primary insomnia had been present between 18 months and 5 years for the three remaining participants. Common for all were erratic sleep patterns, weekend delays of their sleep schedules and naps during school hours. Participants 1 and 3 were both 18-year-old boys whose insomnia was characterized by great difficulties falling asleep. They considered the nighttime worst, although they also described excessive tiredness and sleepiness during daytime. Participants 1 and 3 attended all therapy sessions. Participant 2 was a 16-year-old girl whose insomnia consisted of some troubles falling asleep but also difficulties sleeping as long as she wished. She experienced the daytime consequences of poor sleep as worse than the nighttime symptoms, and was greatly troubled by a variety of mood, cognitive and tiredness symptoms. She fulfilled the criteria of both insomnia and unipolar depression. Depression was considered to be secondary as insomnia had been present for years, and depressive symptoms developed only 2 months before baseline. Participant 2 attended five of the seven therapy sessions and of these one was prolonged to make up for the missed appointments. She did not return a sleep diary at the follow-up measurement.

\section{Procedure}

The participants were assessed with The Duke Structured Interview for Sleep Disorders (DSISD) and The Structured Clinical Interview for DSM-IV (SCID-I). Participants completed the pre-test self-report questionnaires on the same occasion, and they were instructed to start to use a sleep diary the day after. The participants signed an informed consent and APA's ethical guidelines were followed. A random assignment of participants was stratified on the two therapists. All participants started treatment the same week, after the baseline measures. The post-tests were administrated by the therapists on the last session. A follow-up assessment was conducted 3 months after by post.

\section{Measures}

All seven instruments have well established psychometric data. Please refer to the extended report for more details.

Primary outcome measure. Insomnia Severity Index (ISI) was used to assess severity of insomnia. The participants also completed a daily diary with sleep and daytime items.

Process measures. Dysfunctional Beliefs and Attitudes about Sleep Scale (DBAS), Anxiety and Preoccupation about Sleep Questionnaire (APSQ), Pre-Sleep Arousal Scale-C cognitive subscale (PSAS-C), Sleep Associated Monitoring Index (SAMI), and Sleep Related Behaviors Questionnaire (SRBQ) were administered to measure the cognitive processes that the treatment aimed to decrease.

\section{Treatment}

The treatment was carried out by the first and second author and was supervised by the third author. Treatment involved seven, weekly, 1-hour sessions of CT-I. Home assignments were carried out between sessions. The content of treatment was based on Harvey's cognitive model of insomnia and cognitive treatment for insomnia (Harvey, 2002, 2005). A detailed treatment manual was written by the first and second author. Treatment adherence was ensured by the 
use of checklists of therapist interventions that were completed by the therapist after every session.

Session 1. The first session provided an introduction to cognitive therapy and a treatment rationale. A personalized cognitive model over maintaining cognitive processes during the night and the day was derived. The participants set treatment goals.

Session 2. The second session included further work on the personalized model in order to understand the connection between the maintaining processes during the night and the day. An individual treatment plan was made based on which symptoms the participants considered most disturbing, and which processes to target first.

Sessions 3-6. The next sessions focused on attempts to reverse the maintaining processes that were identified during the prior sessions, mainly through behavioural experiments. The participants carried out these experiments both in and between sessions.

Session 7. The last session was devoted to consolidating treatment gains and to prevent relapse. The participants wrote a personalized relapse prevention plan.

\section{Results}

\section{Primary outcome measures}

There was a reduction in scores on the ISI from pre-treatment to post-treatment, and this was retained at the 3-month follow-up. According to the guidelines for scoring and interpretation, participant 1's scores (16) indicated a "clinical insomnia of moderate severity" at pretreatment, "sub threshold insomnia" at post-treatment (8), and "no clinical insomnia" at follow-up (5). Participant 2 reported a clinical insomnia of moderate severity at pre-treatment (18), which had changed to no clinical insomnia at post-treatment (7). This was retained at follow-up (7). The third participant's scores indicated sub threshold insomnia at pre-treatment (13), which changed to no clinical insomnia at post-treatment (5) and at follow-up (5).

Visual inspection showed that Sleep Onset Latency (SOL) was reduced at post-treatment and follow-up for participants 1 and 3, compared to pre-treatment (see Figure 1). This was confirmed by effect size calculations. No change could be confirmed for participant 2 and data are missing for follow-up. Wake after sleep onset (WASO) was very uncommon and short for all three participants. The frequency and length was low at pre-test, post-test and follow-up compared to clinical populations with insomnia. No change of Total Sleep Time (TST) could be confirmed by effect size calculations or visual inspection for any participant (Figure 1).

No stable change could be seen for daytime symptoms for participant 1 over the course of treatment (see Figure 2). Symptoms fluctuated during treatment. At follow-up "irritated, sleepy" and "concentration difficulties" showed a small decline compared to baseline and last week of treatment.

Daytime symptoms data were missing over several periods for participant 2, which makes any description of how symptoms developed during treatment inconclusive (see Figure 2). All daytime symptoms had decreased to lower than baseline for the last week of treatment. Follow-up measures are not available. High fluctuations during baseline and treatment make it difficult to say if improvements are due to treatment or part of a natural development over time.

"Tired, sleepy" and "concentration difficulties" fluctuated greatly at baseline and during treatment for participant 3 . 


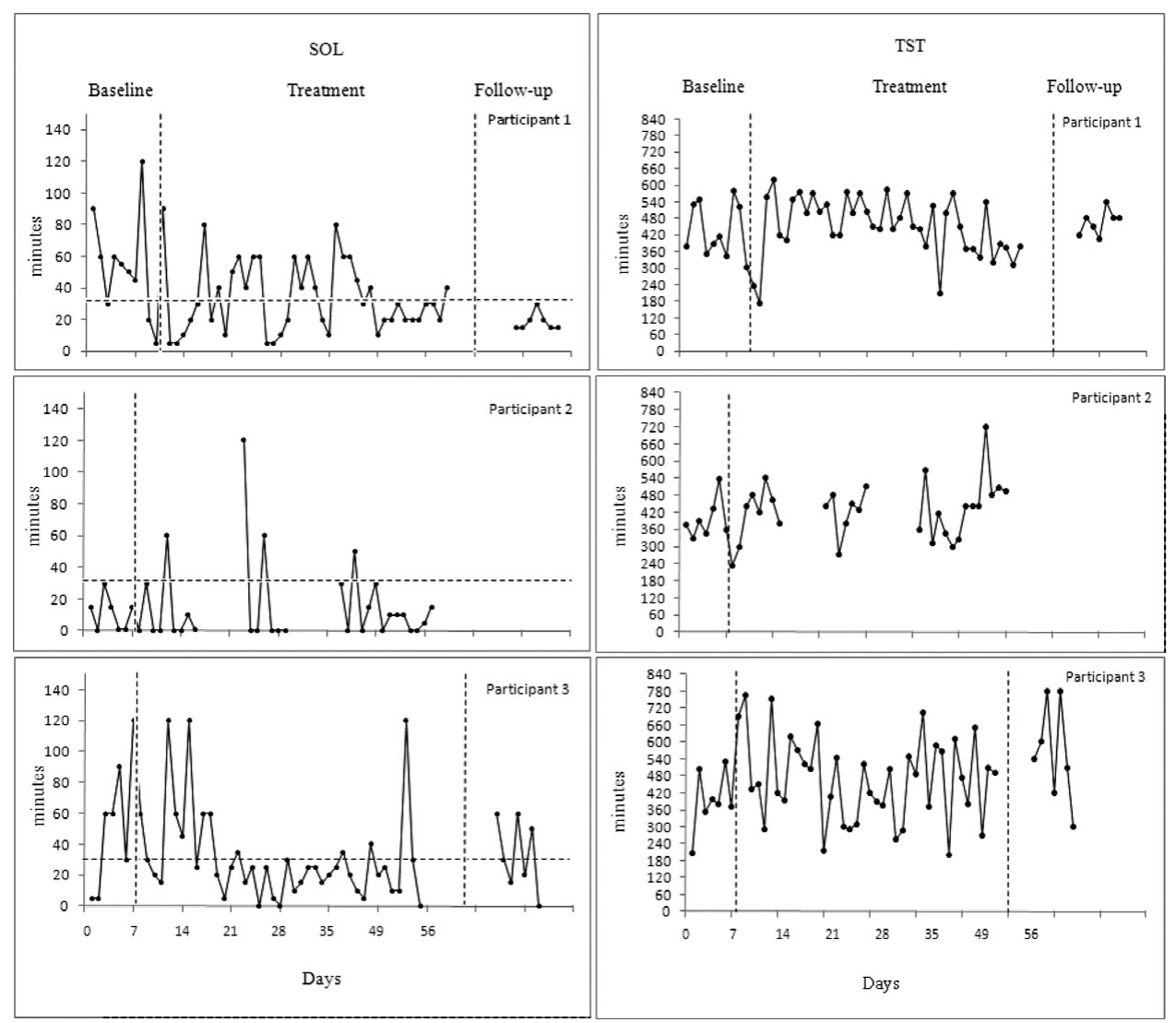

Figure 1. The daily changes in Sleep Onset Latency (SOL) and Total Sleep Time (TST) for the three participants. Cut-off for normal SOL $=30 \mathrm{~min}$ as indicated by the dotted horizontal line.

\section{Insomnia-related processes}

Unhelpful beliefs (DBAS) and sleep-related cognitive arousal (PSAS-C) did not change until follow-up $(-41 \% /-35 \%)$ for participant 1 . Worry (APSQ) and safety behaviours (SRBQ) had decreased at post-treatment $(-22 \% /-21 \%)$ and had decreased further at follow-up. Monitoring (SAMI) was reduced at post-treatment $(-27 \%)$ and was retained at the followup.

Participant 2 had a reduction in scores of unhelpful beliefs at post-treatment $(-44 \%)$ and a rise at follow-up $(-31 \%)$. Worry and monitoring had decreased at post-treatment $(-71 \%$ / $-42 \%)$ and retained a low value at follow-up. Sleep related cognitive arousal $(-42 \%)$ and safety behaviours $(-29 \%)$ decreased at post-treatment and had decreased further at follow-up $(-69 \% /-53 \%)$.

The third participant scored a reduction of unhelpful beliefs at post-treatment $(-18 \%)$. Worry had decreased at post-treatment $(-21 \%)$ but increased at follow-up $(-13 \%)$. Sleep related cognitive arousal had reduced at post-treatment $(-51 \%)$ and retained a low value at 
Tired

Sleepy

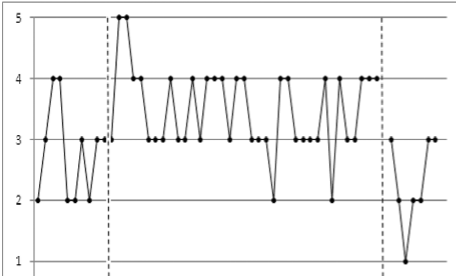

Participant 1
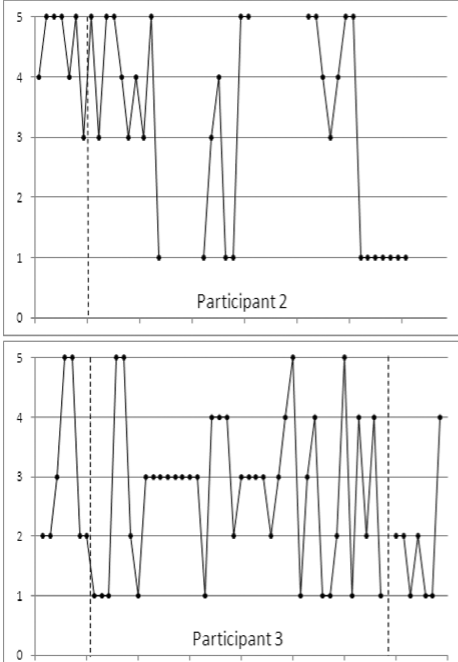

$\begin{array}{lllllll}0 & 7 & 14 & 21 & 28 & 35 & 42\end{array}$
Participant 1

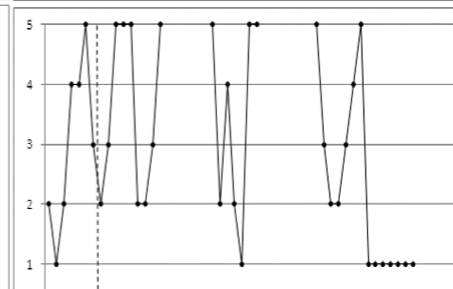

Participant 2

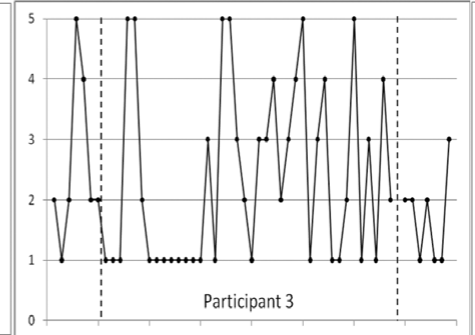

$\begin{array}{lllllll}0 & 7 & 14 & 21 & 28 & 35 & 42\end{array}$
Difficulties concentrating

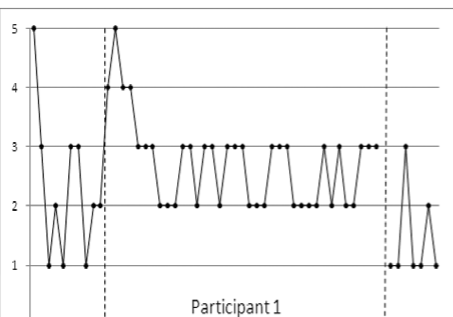

Irritated
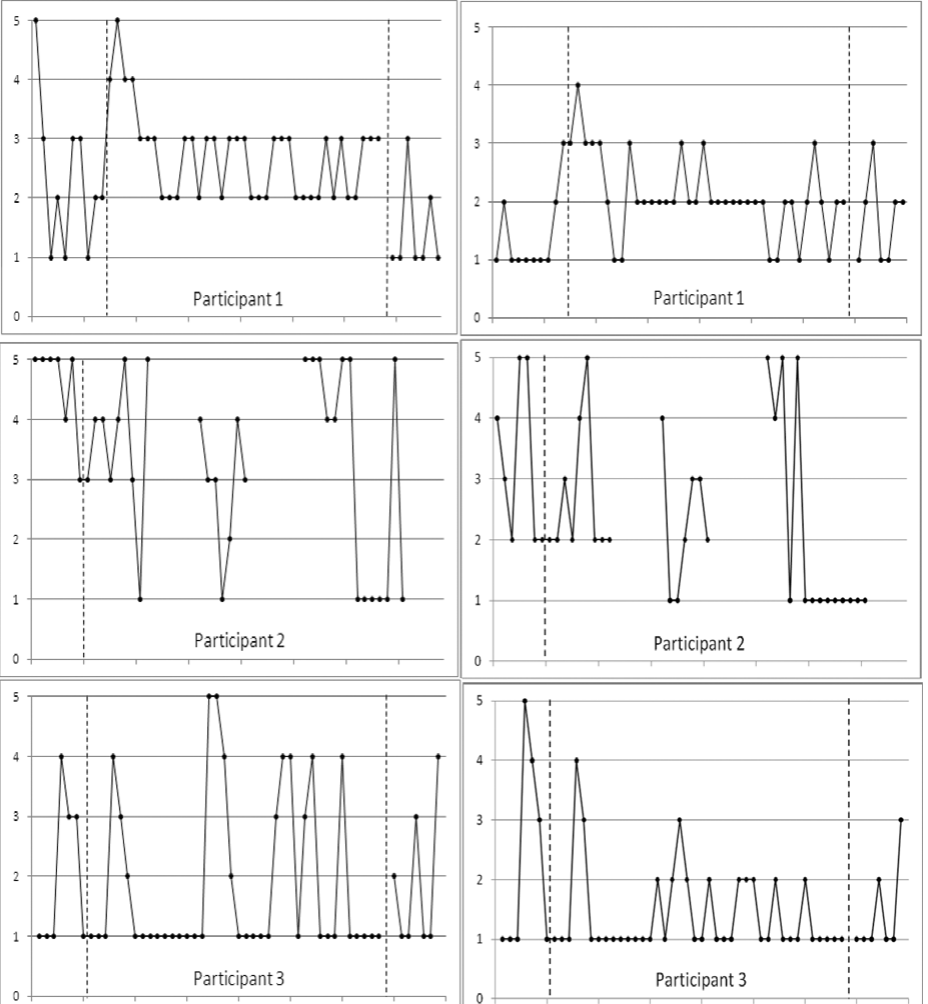

$\begin{array}{lllllll}0 & 7 & 14 & 21 & 28 & 35 & 42\end{array}$

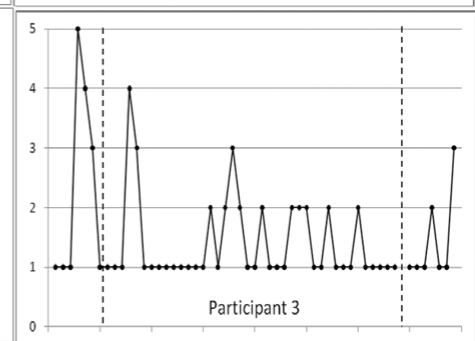

$\begin{array}{lllllll}0 & 7 & 14 & 21 & 28 & 35 & 42\end{array}$

Figure 2. Daily measures of daytime symptoms at baseline, treatment and follow-up. $1=$ not at all $-5=$ a lot. 
follow-up. Safety behaviours were reduced at post-treatment $(-10 \%)$ and retained at followup. A reduction in monitoring was seen at post-treatment $(-52 \%)$ and an increase at follow-up $(-47 \%)$, although still at a large distance from the pre-treatment score.

Please see the extended report for more details on effect size measures, questionnaire data, treatment goals and treatment credibility.

\section{Discussion}

Our aim was to test a psychological insomnia treatment on adolescents and to expand the Cognitive Therapy for Insomnia research to a new age group. The results suggest that adolescents with insomnia, just like adults (Harvey et al., 2007), may benefit from a short cognitive treatment for insomnia. Studies testing treatment options for adolescents are rare and this study takes a much needed step on the way to find solutions to the problem of adolescent insomnia.

An improvement of Sleep Onset Latency was found for participants 1 and 3 but no change of Total Sleep Time (TST) or Wake After Sleep Onset (WASO) could be confirmed. As WASO was low for all participants there was little room for improvement. Sleep diaries showed that TST varied greatly over the treatment period but also within weeks. An explanation could be that lifestyle decisions, rather than inability to sleep, interfered with total sleep time. Daytime symptoms seemingly improved greatly for participant 3 but it is difficult to interpret this result as there were two missing weeks of daily measures. The measure ISI indicated less severe insomnia for all participants.

A surprising finding was that the maintaining processes of insomnia changed to different degrees, or in some cases remained stable, for the participants. This goes against our assumption that less insomnia severity after treatment would mean less maintaining processes. Interpretations are complicated by the lack of cut-off values or normative data available for adolescents with insomnia on these measures. Compared with mean values and SD of the adult participants in a previous CT-I study (Harvey et al., 2007), the participants in the present study had pre-test values on the process measures that were comparable with the first study, although at the higher end within SD, suggesting that the participants in this study had a high degree of maintaining processes. In general, the adolescents had improved less after treatment and at follow-up compared to mean values of the adult participants in the prior CT-I study. Much is unknown about the role of cognitive processes in insomnia. It may be that the cognitive processes have different impact and importance depending on age and that age may moderate how difficult they are to change through treatment. It may also be that some processes are more significant than others when insomnia is developed, maintained, or remitted and change depending on how insomnia has been present or individual differences in the insomnia symptoms. Sleep-related worry, for example, was related to SOL, TST and WASO for people that had suffered from insomnia 7-12 months, but not for shorter insomnia duration, thus indicating that worry is a mechanism that develops a stronger impact over time (Jansson and Linton, 2006). Our research group is currently investigating the roles of the cognitive processes in the development and maintenance of insomnia, in a longitudinal population study (PIPPI: Prospective Investigation of Psychological Processes of Insomnia, unpublished).

The study has some limitations. First, the generality of the findings is limited due to the small number of patients; thus replication is needed. Attrition (two out of five) and missing 
data from one of the remaining participants make it yet harder to draw conclusions about the feasibility of CT-I for adolescents recruited through school, although important variables seem to have changed for those who participated fully in the treatment. Maybe the treatment was considered too long or too time consuming by young people with a busy lifestyle and would have had more appeal if treatment had been shortened or less frequent. It is certainly a challenge to devise a treatment that appeals to a population just about old enough to manage their own healthcare appointments and participation. Another issue is the length of the baseline phase. Participants had irregular sleep habits before treatment and considering the great variability of some daily measures within weeks, a longer baseline would have made the baseline daily measures less vulnerable for possible extreme values. A third issue is that baseline measures, treatments and post-treatment assessment were administrated by the same therapists. It would have been preferable to use an independent researcher for the baseline and post-treatment assessment to limit the risk of social desirability.

Cognitive Therapy for Insomnia may be a promising treatment option for adolescents with insomnia and the treatment needs to be tested further in a randomized controlled trial. More research on insomnia treatments for youth is needed as insomnia often turns into a chronic condition and there is a higher risk of developing health and psychological problems over time (Roberts et al., 2008).

\section{References}

Bootzin, R. R. and Stevens, S. J. (2005). Adolescents, substance abuse, and the treatment of insomnia and daytime sleepiness. Clinical Psychology Review, 25, 629-644.

Breslau, N., Roth, T., Rosenthal, L. and Andreski, P. (1996). Sleep disturbance and psychiatric disorders: a longitudinal epidemiological study of young adults. Biological Psychiatry, 39, 411-418.

Harvey, A. G. (2002). A cognitive model of insomnia. Behavior Research and Therapy, 40, 869-893.

Harvey, A. G. (2005). A cognitive theory and therapy for chronic insomnia. Journal of Cognitive Psychotherapy, 19, 41-59.

Harvey, A. G., Sharpley, A. L., Ree, M. J., Stinson, K. and Clark, D. M. (2007). An open trial of cognitive therapy for chronic insomnia. Behaviour Research and Therapy, 45, 2491-2501.

Jansson, M. and Linton, S. J. (2006). The development of insomnia within the first year: a focus on worry. British Journal of Health Psychology, 11, 501-511.

Morin, C. M., Bootzin, R. R., Buysse, D. J., Edinger, J. D., Espie, C. A. and Lichstein, K. L. (2006). Psychological and behavioral treatment of insomnia: an update of recent evidence (19982004). Sleep, 29, 1396-1406.

Moseley, L. and Gradisar, M. (2009). Evaluation of a school-based intervention for adolescent sleep problems. Sleep, 32, 334-341.

Roberts, R. E., Roberts, C. R. and Duong, H. T. (2008). Chronic insomnia and its negative consequences for health and functioning of adolescents: a 12-month prospective study. Journal of Adolescent Health, 42, 294-302.

Sousa, I. C. D., Araújo, J. F. and Azevedo, C. V. M. D. (2007). The effect of a sleep hygiene education program on the sleep-wake cycle of Brazilian adolescent students. Sleep and Biological Rhythms, 5, $251-258$. 